\title{
AUFSÄTZE
}

\section{Kann man sich auf Karlsruhe verlassen? Eine kritische Bestandsaufnahme am Beispiel des Wahlrechts*}

\author{
Gerd Strohmeier
}

Die Deutsche Meisterschaft in der Fußballbundesligasaison 1999/2000 wurde relativ knapp entschieden: Auf Platz 1 der Abschlusstabelle stand der FC Bayern München mit 73 Punkten, auf Platz 2 Bayer Leverkusen, ebenfalls mit 73 Punkten - jedoch mit einer schlechteren Tordifferenz (+38) im Vergleich zum FC Bayern $(+45)^{1}$, die bei Punktgleichheit den Ausschlag gibt. ${ }^{2}$ Allerdings lag der FC Bayern nicht nur wegen der besseren Tordifferenz, sondern auch wegen der wenige Jahre zuvor eingeführten und seit der Saison 1995/1996 praktizierten „Drei-Punkte-Regel“ 3 auf dem ersten Rang. Nach Anwendung der klassischen „Zwei-Punkte-Regel“ wäre in der Fußballbundesligasaison 1999/2000 eine andere Mannschaft Deutscher Meister geworden: Bayer Leverkusen mit 52:16 Punkten, vor dem FC Bayern München mit 51:17 Punkten. An diesem Beispiel lässt sich verdeutlichen, dass Ergebnisse von Regelwerken ein Stück weit präjudiziert werden. Dies gilt natürlich nicht nur im Sport, sondern auch in der Politik, zum Beispiel im Bereich des Wahlrechts - selbstverständlich mit einer völlig anderen Tragweite und Bedeutung.

„Wer das Wahlrecht reformiert“, formuliert Henner Jörg Boehl zutreffend, „der operiert (bildlich gesprochen) an der Herzkammer der Demokratie“ ${ }^{\text {, }}$, da das Wahlrecht das Regelwerk bildet, unter dem (repräsentative) Demokratie erst möglich beziehungsweise praktisch umgesetzt wird. ${ }^{5}$ Da sich Volksherrschaft bei Wahlen im Wahlergebnis manifestiert und das Wahlrecht dieses Ergebnis nicht unwesentlich präjudiziert, tragen diejenigen, die über das Wahlrecht entscheiden, die also (um im Bild zu bleiben) an der Herzkammer der Demokratie operieren, sehr große Verantwortung - ebenso wie jene, die den Rahmen für diese Entscheidung schaffen; im Bild: die entscheiden, wo, wie und mit welchen Mitteln operiert wird. Von herausragender Bedeutung ist dabei unter anderem die Frage, inwiefern sich derjenige, der

* Der Beitrag basiert in wesentlichen Teilen auf einer Stellungnahme, die der Autor als Sachverständiger für die öffentliche Anhörung des Innenausschusses des Deutschen Bundestages am 14. Januar 2013 abgegeben hat (A-Drs. 17(4)634 H).

1 Vgl. Abschlusstabelle der Bundesliga 1999/2000, http://www.fussballdaten.de/bundesliga/2000 (Abruf am 12. April 2013).

2 Vgl. $\$ 24$ Nr. 1 c DFB Spielordnung 1999/2000.

3 Vgl. \$24 Nr. 1 a DFB Spielordnung 1999/2000.

4 Henner Jörg Boehl, Wahlrecht und Volksparteien, in: Ralf Thomas Baus (Hrsg.), Parteiensystem im Wandel. Perspektiven, Strategien und Potentiale der Volksparteien, Sankt Augustin / Berlin 2013, S. 121 - 137, S. 122.

5 Infolgedessen hat das Bundesverfassungsgericht die konkrete Ausgestaltung des Wahlsystems im Bundeswahlgesetz zu Recht als „materielles Verfassungsrecht“ (vgl. BVerfG, 2 BvC 1/07, 2 BvC 7/07 vom 3. Juli 2008, Rn. 94) und Jörn Ipsen das Bundeswahlgesetz als das wichtigste Gesetz unterhalb der Verfassungsebene bezeichnet (vgl. ders., Wahlrecht im Spannungsfeld von Politik und Verfassungsgerichtsbarkeit, in: DVBl, 128. Jg. (2013), H. 5, S. 265 - 274, S. 265). 
über das Wahlrecht entscheidet - der Gesetzgeber - auf denjenigen verlassen kann, der die Rahmenbedingungen für diese Entscheidung setzt - das Bundesverfassungsgericht (BVerfG). Dieser Frage soll am Beispiel der Verfassungsrechtsprechung zu Überhangmandaten, dem Effekt des negativen Stimmgewichts ${ }^{6}$ und dem Wahlrecht von Auslandsdeutschen nachgegangen werden.

\section{1. Überhangmandate}

Seit 1957 werden (ausgleichslose) Überhangmandate durch das BVerfG - unter bestimmten Voraussetzungen und innerhalb bestimmter Grenzen - verfassungsrechtlich anerkannt. ${ }^{7}$ In seinem Urteil vom 10. April 1997 wies das Gericht selbst darauf hin, dass es „in ständiger Rechtsprechung festgestellt " habe, dass die Bundestagswahl „den Grundcharakter einer Verhältniswahl“ trage und Überhangmandate den „Erfolgswert der Wählerstimmen“ differenzierten, diese „Differenzierung [...] aber als die notwendige Folge des besonderen Charakters der personalisierten Verhältniswahl mit der Wahlgleichheit [...] vereinbar [sei], solange die Wahlkreise im Rahmen des Möglichen annähernd gleich groß seien" ${ }^{8}$. Zugleich hat es aber auch zum wiederholten Male darauf hingewiesen, dass der Gesetzgeber darauf achten müsse, „dass sich die Zahl der Überhangmandate in Grenzen hält" ". Während diese Komponenten Konstanten der Verfassungsrechtsprechung zu Überhangmandaten bilden, hat sich das BVerfG im Laufe der Zeit höchst unterschiedlich zu der Frage geäußert, wo die Grenze für die Zahl der Überhangmandate verläuft: Am 3. Juli 1957 hat es von „engen Grenzen“10 gesprochen, diese aber nicht weiter konkretisiert. Am 22. Mai 1963 hat es darauf hingewiesen, dass die Grenzen nicht überschritten würden, wenn alle Wahlkreise „etwa gleich groß "11 seien. Am 24. November 1988 hat es erneut von „engen Grenzen“ gesprochen, ohne diese weiter zu konkretisieren - jedoch auf die Stimmenzahlen verwiesen, die die Parteien bei der Bundestagswahl 1987 durchschnittlich für die Erlangung eines Mandats benötigten, und hervorgehoben, dass die CDU, die ein Überhangmandat gewonnen hatte, damit ,immer noch zwischen der Partei mit der geringsten [...] und der höchsten [...] Stimmenzahl für ein Mandat"12 liege.

Am 10. April 1997 machte das BVerfG allerdings deutlich, dass sich seiner bisherigen Rechtsprechung nicht entnehmen lasse, „dass Überhangmandate den Grundcharakter der Bundestagswahl als Verhältniswahl nur insofern modifizieren dürften, als sich die [daraus resultierende] Verstärkung des Gewichts [...] im Rahmen der durch das mathematische Sitzverteilungsverfahren ohnehin vorgegebenen und unvermeidlichen Differenzierung hält“13. In diesem Zusammenhang wies es darauf hin, dass es bereits in seinem Beschluss

6 Dieser sollte besser als Effekt des inversen Erfolgswerts bezeichnet werden (vgl. dazu Gerd Strohmeier, Vergangene und zukünftige Reformen des deutschen Wahlsystems, in: ders. (Hrsg.), Wahlsystemreform, ZPol-Sonderheft 19, Baden-Baden 2009, S. 11 - 44, S. 27). Allerdings wird der Effekt gemeinhin als Effekt des negativen Stimmgewichts etikettiert.

7 Vgl. BVerfG, 2 BvR 9/56, 3. Juli 1957.

8 BVerfG, 2 BvF 1/95 vom 10. April 1997, Rn. 92.

9 Ebenda.

10 BVerfG, 2 BvR 9/56 vom 3. Juli 1957, Rn. 31.

11 BVerfG, 2 BvC 3/62 vom 22. Mai 1963, Rn. 26.

12 BVerfG, BvC 4/88 vom 24. November 1988, Rn. 16.

13 BVerfG, 2 BvF 1/95 vom 10. April 1997, Rn. 84. 
vom 22. Mai 1963 „[e]ine deutlich stärkere Differenzierung des Stimmgewichts durch Überhangmandate“ 14 gebilligt habe und bislang „keinerlei Anlass [gehabt hätte], die Grenzen der Zulässigkeit von Überhangmandaten näher zu bestimmen“15. Diesmal wurde das Gericht jedoch konkreter: So seien die Grenzen überschritten, wenn „Überhangmandate von Wahl zu Wahl regelmäßig in größerer Zahl anfallen “16, und möge bei der Definition der größeren Zahl „[d] as Fünfprozentquorum [...] - bezogen auf die reguläre Gesamtzahl der Parlamentssitze - als Anhalt dienen“ 17 . Zugleich betonten die Richter aber, dass sie „das Überhangmandat verfassungsrechtlich anerkannt [hätten], auch wenn sich darauf eine Mehrheit im Bundestag und die Wahl einer Bundesregierung gründen sollte “18, und es „in erster Linie Sache des Gesetzgebers“ sei, „[z] u befinden, ob Anlass für eine Änderung des Wahlrechts besteht“ und „zu bestimmen, in welchem Ausmaß eine Erhöhung der Sitzzahl nach der in $\$ 6$ Abs. 5 BWG getroffenen Regelung [durch Überhangmandate] hinzunehmen ist"19. Damit war die Bestimmung der Grenze primär eine Entscheidung des Gesetzgebers und nicht des Bundesverfassungsgerichts; das vom Gericht genannte - und vor dem Hintergrund der Sperrklausel nachvollziehbar entwickelte - Fünf-Prozent-Quorum sei zudem keine verbindliche „Obergrenze“, sondern ein „Orientierungspunkt“20 für den Gesetzgeber. Diese Entscheidung ist zwar nur von vier Richtern getragen ${ }^{21}$, jedoch in der einstimmig ergangenen Entscheidung vom 3. Juli 2008 nicht korrigiert worden, in der das BVerfG ausschließlich den Effekt des negativen Stimmgewichts beanstandet ${ }^{22}$ und zu den Überhangmandaten „,ausdrücklich geschwiegen “ 23 und diese damit „nach wie vor akzeptiert" ${ }^{24}$ hat.

Am 26. Februar 1998 entschieden die Karlsruher Richter allerdings, dass eine Landesliste Ersatzleute „nur im Rahmen der Abgeordnetenzahl bereit [hält], die aufgrund des Zweitstimmenergebnisses für die Landesliste ermittelt worden ist " ${ }^{25}$, und infolgedessen das Sitzkontingent der Landesliste erschöpft ist, „[s]olange die Partei des weggefallenen Wahlkreisabgeordneten in dem betreffenden Land über Überhangmandate verfügt "26. Dieses Urteil war sicherlich nicht nur für die breite Öffentlichkeit ${ }^{27}$, sondern auch für die Fachöffentlichkeit und den Gesetzgeber überraschend. Schließlich ist es erstaunlich, „dass das Bundesverfassungsgericht auf diesen Gedanken erst verfallen ist, nachdem nahezu fünf Jahrzehnte die

14 Ebenda, Rn. 85.

15 Ebenda, Rn. 102.

16 Ebenda, Rn. 101.

17 Ebenda, Rn. 102.

18 Ebenda, Rn. 82.

19 Ebenda, Rn. 102.

20 Ebenda.

21 Nach $\$ 15$ Abs. 4 Satz 3 BVerfGG kann bei Stimmengleichheit, die in diesem Fall mit einer Vierzu-vier-Entscheidung vorlag, ,ein Verstoß gegen das Grundgesetz oder sonstiges Bundesrecht nicht festgestellt werden“. Im Übrigen kann letztlich „nur maßgeblich sein, was Aussage einer getroffenen Entscheidung - mit welcher Mehrheit auch immer - war" (Volker M. Haug, Das Bundesverfassungsgericht als Gesetzgeber anstelle des Gesetzgebers: Ein kritischer Blick auf das Wahlrechtsurteil vom 25. Juli 2012, in: ZParl, 43. Jg. (2012), H. 3, S. 658 - 674, S. 670).

22 Vgl. BVerfG, 2 BvC 1/07, 2 BvC 7/07 vom 3. Juli 2008.

23 Volker M. Haug, a.a.O. (Fn. 21), S. 671.

24 Jörn Ipsen, a.a.O. (Fn. 5), S. 269.

25 BVerfG, 2 BvC 28/96 vom 26. Februar 1998, Ls $3 a$.

26 Ebenda, Ls 3b.

27 Vgl. Jörn Ipsen, a.a.O. (Fn. 5), S. 267. 
Listennachfolge in Direktmandate völlig unbestritten war - gleichgültig, ob für die Partei in dem betreffenden Land Überhangmandate angefallen waren oder nicht ${ }^{\text {" } 28}$. Ob durch diese Kehrtwende - wie Jörn Ipsen meint - das Urteil vom 10. April 1997 teilweise revidiert bezie-

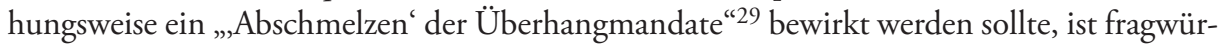
dig. Dennoch wird dadurch eine kritische(re) Haltung zu Überhangmandaten deutlich - die allerdings nicht überzeugend begründet werden konnte und in gewisser Weise inkonsequent erscheint: „Wenn es einerseits verfassungsmäßig sein soll, dass Überhangmandate (auch) eine Regierungsbildung ermöglichen, so wird man den Mandaten - die ja als ,Überhangmandate nicht erkennbar sind - nicht die Legitimität absprechen können, auch auf Dauer - und gegebenenfalls durch Listennachfolge - im Parlament vertreten zu sein. "30

Am 25. Juli 2012 vollzog das BVerfG jedoch eine deutliche Abkehr von seinem Urteil vom 10. April 1997. Danach liegt die Entscheidung, ob aufgrund der Überhangmandate das Wahlrecht geändert werden muss beziehungsweise in welchem Umfang Überhangmandate hinzunehmen sind, nicht mehr primär beim Gesetzgeber: „Das Bundeswahlgesetz [...] nimmt verschiedene Anliegen in sich auf. Zwar obliegt der Ausgleich dieser Anliegen in erster Linie der politischen Willensbildung im Gesetzgebungsverfahren, es ist jedoch im speziellen Zusammenhang Aufgabe des Bundesverfassungsgerichts, gleichheitsrechtliche Anforderungen an das Sitzzuteilungssystem so zu konkretisieren, dass der Gesetzgeber das Wahlrecht auf verlässlicher verfassungsrechtlicher Grundlage gestalten kann. "31 Zudem verwarf das Gericht das Fünf-Prozent-Quorum als Orientierungspunkt für die Grenze der Zahl der Überhangmandate, wobei es, ohne Umschweife zu machen, selbst auf seinen Bruch mit dem Urteil vom 10. April 1997 hinwies: „Fallen sie [die Überhangmandate] regelmäßig in größerer Zahl an, widerspricht dies der Grundentscheidung des Gesetzgebers [...]. Wann dies der Fall ist, lässt sich - entgegen der Ansicht der die Entscheidung vom 10. April 1997 tragenden Richter [...] - nicht allein in Orientierung an dem Fünf-Prozent-Quorum [...] bestimmen. "32 Schließlich halbierte das Gericht das Fünf-Prozent-Quorum: „Die Wahlrechtsgleichheit und die Chancengleichheit der Parteien sind bei einem Anfall ausgleichsloser Überhangmandate im Umfang von mehr als etwa einer halben Fraktionsstärke verletzt. "33 Allerdings ist darauf hinzuweisen, dass das Urteil vom 25. Juli 2012 in diesem Punkt unpräzise ist. So ist einmal von „etwa einer halben Fraktionsstärke “34 und an anderer Stelle „von 15 Überhangmandaten“35 die Rede, was sich natürlich nur bei einer Gesamtsitzzahl von 598 Mandaten deckt. ${ }^{36}$ Weiterhin machte das BVerfG aus dem „Orientierungspunkt" des Gesetzgebers eine verbindliche „Obergrenze“ der Verfassung(srechtsprechung) - eine „verfassungsrechtliche Grenze für die ausgleichslose Zuteilung von Überhangmandaten " 37 . Angesichts der oben aufgeführten früheren Rechtsprechung zu Überhangmandaten kann das Urteil vom 25. Juli 2012 nur über-

28 Ebenda, S. 268.

29 Ebenda, S. 267.

30 Ebenda, S. 268.

31 BVerfG, 2 BvF 3/11, 2 BvR 2670/11, 2 BvE 9/11 vom 25. Juli 2012, Rn. 144.

32 Ebenda, Rn. 141.

33 Ebenda, Rn. 140.

34 Ebenda, Rn. 127, Rn. 140.

35 Ebenda, Rn. 144; vgl. auch Rn. 145.

36 Insgesamt wird jedoch deutlich, dass im Entscheidungsfall nicht die absolute Zahl von 15, sondern die prozentuale Angabe von einer halben Fraktionsstärke die verbindliche „Obergrenze“ markiert.

37 Ebenda, Rn. 127. 
raschen und als Kehrtwende bezeichnet werden, denn es hat „offen mit der bisherigen Rechtsprechungslinie in einer politisch bedeutsamen und verfassungsrechtlich sensiblen Frage“ 38 gebrochen. Die Argumentation des Gerichts, dass „[i]m Hinblick auf die Notwendigkeit, den Wahlen zu den kommenden Bundestagen eine verlässliche rechtliche Grundlage zu geben und diese nicht dem Risiko einer Auflösung im Wahlprüfungsverfahren wegen Fehlens erforderlicher Regelungen auszusetzen, die vorliegenden gesetzlichen Wertungen in einem handhabbaren Maßstab zusammenzuführen [seien], an den der Gesetzgeber anknüpfen kann" ${ }^{39}$, ist nicht nachvollziehbar, weil ein handhabbarer Maßstab bereits existierte, an den der Gesetzgeber anknüpfen konnte, aber eben bislang nicht musste.

Begründet hat das BVerfG diese Kehrtwende zum einen mit dem angeblichen Willen des Gesetzgebers. Schließlich habe dieser bei der Verabschiedung des Neunzehnten Gesetzes zur Änderung des Bundeswahlgesetzes mit der Regelung zu den Berliner Zweitstimmen ${ }^{40}$ „seinen Willen bekräftigt, den Einfluss der Erststimme auf die Verteilung der Listenmandate einzudämmen“, und mit der vorrangigen Zuweisung der (aus der Reststimmenverwertung resultierenden) Zusatzmandate an Landeslisten mit Überhangmandaten „unterstrichen, dass die Zahl der proporzwidrigen Überhangmandate zu minimieren ist “41. Dies ist schlichtweg unzutreffend, da der Gesetzgeber die Regelung zu den Berliner Zweitstimmen vom BVerfG letztlich aufgetragen bekam ${ }^{42}$ und die vorrangige Zuweisung der Zusatzmandate an Landeslisten mit Überhangmandaten allein der Vermeidung des Effekts des negativen Stimmgewichts diente. Als weiterer Grund wurde angeführt, dass „zu erwarten [sei], dass die Zahl der Überhangmandate den hinnehmbaren Umfang auf absehbare Zeit deutlich übersteigen wird“43. Dies ist möglicherweise richtig und durchaus relevant für das Erreichen des „hinnehmbaren Umfangs“ beziehungsweise der „Obergrenze“, aber kein Grund zum Verschieben der „Obergrenze“. Diese lag bei der Bundestagswahl 2009 bei 30 Überhangmandaten und wurde mit 24 nicht (auch nicht beinahe) erreicht ${ }^{44}$; zudem wäre ihr Erreichen in Zukunft durch die vorrangige Zuweisung der Zusatzmandate an Landeslisten mit Überhangmandaten $^{45}$ - wie das BVerfG richtig erkannt hat - erschwert worden. ${ }^{46}$ Dass die neue „Obergrenze“ - wie das Gericht weiterhin feststellte - „der vom Senat im Urteil vom 10. April 1997 gebilligten Quote von [bei der Bundestagswahl 1994 erreichten] 16 Überhangmandaten bei einer regulären Abgeordnetenzahl von 656“47 entspricht, zeigt den Versuch der Richter,

38 Volker M. Haug, a.a.O. (Fn. 21), S. 670.

39 BVerfG, 2 BvF 3/11, 2 BvR 2670/11, 2 BvE 9/11 vom 25. Juli 2012, Rn. 140.

40 Vgl. dazu ausführlich Gerd Strohmeier, a.a.O. (Fn. 6), S. 38.

41 BVerfG, 2 BvF 3/11, 2 BvR 2670/11, 2 BvE 9/11 vom 25. Juli 2012, Rn. 142.

42 Vgl. BVerfG, 2 BvC 4/04 vom 15. Januar 2009, Rn. 28; Heinrich Lang, in: A-Drs. 16(4)592 F, S. 11.

43 BVerfG, 2 BvF 3/11, 2 BvR 2670/11, 2 BvE 9/11 vom 25. Juli 2012, Rn. 146.

44 Vgl. auch Volker M. Haug, a.a.O. (Fn. 21), S. 671.

45 Auf diese Weise wären einige Überhangmandate mit Zweitstimmen unterlegt worden, wodurch sie - wie es Jörn Ipsen, a.a.O. (Fn. 5), S. 270 ausgedrückt hat - ihre „Qualität als Überhangmandat“ verloren hätten.

46 Nach Hesse hätte die Reststimmenverwertung des Neunzehnten Gesetzes zur Änderung des Bundeswahlgesetzes die Überhangmandate im Durchschnitt um etwa 25 Prozent verringert (vgl. Christian Hesse, Wahlrecht 2013 mit Schattenseiten. Oder: Ein kritisches Lob dem vom Bundesverfassungsgericht verworfenen Bundeswahlgesetz von 2011, in: ZParl, 44. Jg. (2009), H. 1, S. $177-200$, S. 184).

47 BVerfG, 2 BvF 3/11, 2 BvR 2670/11, 2 BvE 9/11 vom 25. Juli 2012, Rn. 143. 
„einen Kompromiss zwischen früherer und derzeitiger Rechtsprechung “48 anzudeuten, vermag aber nicht einmal ansatzweise den Bruch zwischen dem 1997er- und dem 2012erUrteil zu kaschieren ${ }^{49}$; es „täuscht lediglich eine Kontinuität in der Rechtsprechung vor “50. Wie sich die neue „Obergrenze“ für Überhangmandate herleiten beziehungsweise begründen lässt, weiß indes niemand ${ }^{51}$ - nicht einmal das Bundesverfassungsgericht selbst, das, ohne Umschweife zu machen, „[i]n ungewohnter Offenheit“52, einräumte, „dass die Zahl von 15 Überhangmandaten als Akt richterlicher Normkonkretisierung nicht vollständig begründet werden kann “53. Infolgedessen kann das Urteil verfassungsrechtlich nicht überzeugen, wenngleich es „dem ,Mainstream ' der öffentlichen wahlrechtspolitischen Debatte“54 weitgehend entsprechen dürfte. Schließlich sind seine Begründungen auch „wesentlich mehr politisch als juristisch anmutend" 55 .

Unabhängig von der Begründung der neuen „Obergrenze“ für Überhangmandate stand für das BVerfG fest, dass die bei der Bundestagswahl 2009 erreichte und in Zukunft zu erwartende „Zahl an Überhangmandaten [...] deutlich oberhalb der [neuen] zulässigen Höchstgrenze“ liege und „nunmehr eine Handlungspflicht des Gesetzgebers" 56 bestehe womit dessen Handlungsspielraum nicht unmaßgeblich eingeschränkt wurde, auch wenn keine komplette Vermeidung beziehungsweise kein vollständiger Ausgleich von Überhangmandaten gefordert wurde. Das Bundesverfassungsgericht hat damit nicht - wie Volker $M$. Haug meint - „den gesetzgeberischen Ermessensspielraum auf Null“ 57 reduziert, aber doch erheblich begrenzt. Alles in allem handelt es sich - wie Haug richtig bilanziert - um einen weitreichenden Eingriff beziehungsweise „äußerst fragwürdigen Übergriff des BVerfG in die Domäne des Gesetzgebers" 58 .

\section{Effekt des negativen Stimmgewichts}

Der Effekt des negativen Stimmgewichts (NSG) - das heißt im Wesentlichen der Effekt, dass ein Zuwachs an Stimmen einer Partei zu einem Verlust an Sitzen oder ein Verlust an Stimmen einer Partei zu einem Zuwachs an Sitzen derselben Partei führen kann - ist dem Bundesverfassungsgericht lange Zeit vor seinem „NSG-Urteil“ vom 3. Juli 2008 bekannt gewesen und nicht von diesem beanstandet worden:

48 Niels Dehmel / Eckhard Jesse, Das neue Wahlgesetz zur Bundestagswahl 2013. Eine Reform der Reform der Reform ist unvermeidlich, in: ZParl, 44. Jg. (2013), H. 1, S. 201 - 213, S. 205. Letztlich war das Bundesverfassungsgericht weder bereit, ,an seine frühere Rechtsprechung zu den Überhangmandaten anzuknüpfen“, noch „ein klares Bekenntnis zur Diskontinuität abzulegen“ (Jörn Ipsen, a.a.O. (Fn. 5), S. 271).

49 Vgl. Volker M. Haug, a.a.O. (Fn. 21), S. 670.

50 Jörn Ipsen, a.a.O. (Fn. 5), S. 272.

51 Letztlich werden - wie Ipsen richtig darstellt - durch das Urteil „mehr Fragen aufgeworfen als Antworten gegeben" (ebenda).

52 Volker M. Haug, a.a.O. (Fn. 21), S. 669.

53 BVerfG, 2 BvF 3/11, 2 BvR 2670/11, 2 BvE 9/11 vom 25. Juli 2012, Rn. 144.

54 Volker M. Haug, a.a.O. (Fn. 21), S. 658.

55 Ebenda, S. 670.

56 BVerfG, 2 BvF 3/11, 2 BvR 2670/11, 2 BvE 9/11 vom 25. Juli 2012, Rn. 145.

57 Volker M. Haug, a.a.O. (Fn. 21), S. 670.

58 Ebenda. 
Im Urteil vom 10. April 1997 war nichts diesbezügliches zu lesen, obwohl das NSG von der niedersächsischen Landesregierung eindeutig beklagt und hinlänglich beschrieben wurde: „Die inkonsequente Ausgestaltung des geltenden Rechts führe zu unsinnigen Ergebnissen bei der Sitzzuteilung. Sie ermögliche etwa, dass eine Partei umso mehr Mandate gewinne, je weniger Stimmen sie erhalte. "59 Dies wurde seitens des Bundesverfassungsgerichts aber ebenso wenig beanstandet wie (im Grundsatz) die Überhangmandate. Damit war davon auszugehen, dass der Effekt des NSG - genauso wie die Differenzierung des Erfolgswerts der Wählerstimmen durch Überhangmandate - als eine „notwendige Folge des besonderen Charakters der personalisierten Verhältniswahl" 60 betrachtet werden musste.

Am 16. Juni 2000 teilte das BVerfG dem Deutschen Bundestag im Rahmen des Berichterstatterschreibens des Richters Hans-Joachim Jentsch mit, dass der „Umstand, dass eine[r] Partei [...] deshalb mehr Sitze zuzuteilen sein können, weil sie in bestimmten Ländern weniger Stimmen erhalten habe, [...] in der mündlichen Verhandlung vor dem Bundesverfassungsgericht am 19. November 1996 im Verfahren 2 BvF 1/95 erörtert worden "61 sei und das Gericht in seiner Entscheidung hierzu ausdrücklich Folgendes festgestellt habe: „Das geltende Bundeswahlgesetz leistet Gewähr, dass jeder Wähler mit seiner Erst- und Zweitstimme und deren Zusammenwirken die gleiche rechtliche Möglichkeit hat, auf das Wahlergebnis Einfluss zu nehmen." 62

Am 22. Januar 2001 verwarf das BVerfG Wahlprüfungsbeschwerden zur Bundestagswahl 1998, die den Effekt des NSG zum Gegenstand hatten, als „offensichtlich unbegründet“63.

Am 3. Juli 2008 kam das Bundesverfassungsgericht jedoch zu dem Ergebnis, dass der Effekt des NSG „gegen die Grundsätze der Gleichheit und der Unmittelbarkeit der Wahl“64 verstoße - den zuerst genannten Grundsatz sogar „in eklatanter Weise“65 beeinträchtige. Im Tenor stellte das Gericht fest, dass „\$7 Absatz 3 Satz 2 in Verbindung mit $\$ 6$ Absätze 4 und 5 des Bundeswahlgesetzes [...] Artikel 38 Absatz 1 Satz 1 des Grundgesetzes [verletzt], soweit hierdurch ermöglicht wird, dass ein Zuwachs an Zweitstimmen zu einem Verlust an Sitzen der Landeslisten oder ein Verlust an Zweitstimmen zu einem Zuwachs an Sitzen der Landeslisten führen kann"66. In der Urteilsbegründung fügte das Gericht diesem klassischen Tatbestand einen weiteren hinzu: „Ein Wahlsystem, das darauf ausgelegt ist oder doch jedenfalls in typischen Konstellationen zulässt, dass ein Zuwachs an Stimmen zu Mandatsverlusten führt oder dass für den Wahlvorschlag einer Partei insgesamt mehr Mandate erzielt werden, wenn auf ihn selbst weniger [klassischer Tatbestand] oder auf einen konkurrierenden Vorschlag [erweiterter Tatbestand] mehr Stimmen entfallen, führt zu willkürlichen Ergebnissen und lässt den demokratischen Wettbewerb um Zustimmung bei den Wahlberechtigten widersinnig erscheinen. "67 Zudem sprach es an dieser Stelle nur noch von Stimmen, nicht mehr

59 BVerfG, 2 BvF 1/95 vom 10. April 1997, Rn. 43.

60 Ebenda, Rn. 170.

61 Hans-Joachim Jentsch, zitiert in: Heinrich Lang, in: A-Drs. 16(4)592 F, S. 3.

62 Ebenda.

63 BVerfG, 2 BvC 1/99 und 5/99 vom 22. Januar 2001.

64 BVerfG, 2 BvC 1/07, 2 BvC 7/07 vom 3. Juli 2008, Rn. 76.

65 Ebenda, Rn. 103.

66 Ebenda, Tenor Nr. 1.

67 Ebenda, Rn. 103. Während der klassische NSG-Tatbestand einen unilateralen Effekt darstellt, handelt es sich bei dem erweiterten NSG-Tatbestand um einen bilateralen Effekt (vgl. Christian Hesse, a.a.O. (Fn. 46), S. 187). 
ausschließlich von Zweitstimmen. Allerdings hat es überwiegend auf die Zweitstimmen abgestellt ${ }^{68}$, und es lässt sich dem Kontext entnehmen, dass Zweitstimmen gemeint sein müssen. ${ }^{69}$ Festzuhalten ist allerdings, dass die Definition des NSG unpräzise und in der Folge interpretationsbedürftig war. Unabhängig davon kann das Urteil vom 3. Juli 2008 angesichts der oben aufgeführten früheren Rechtsprechung des Bundesverfassungsgerichts zum Effekt des NSG - wie das Urteil vom 25. Juli 2012 - nur überraschen und als Kehrtwende bezeichnet werden. Zudem ist die dort festgestellte Verletzung der Gleichheit der Wahl partiell unzutreffend beziehungsweise unbegründet, da der Effekt des NSG die Erfolgswertgleichheit nicht nur reduzieren, sondern auch erhöhen kann - nämlich dann, wenn mehr Stimmen für eine Partei zu weniger Sitzen dieser Partei führen. ${ }^{70}$ Grundsätzlich fragwürdig ist indessen die vom BVerfG festgestellte Verletzung der Unmittelbarkeit der Wahl, da der Effekt des NSG beziehungsweise dessen Begleiterscheinungen „nichts mit der Unmittelbarkeit zu tun "71 haben, die im Kern nur fordert, „dass zwischen Wähler und zu bildender Repräsentation kein Gremium zwischengeschaltet sein darf "72. So sehr der Effekt des NSG auch politisch als ungewünschte Erscheinung der personalisierten Verhältniswahl gesehen werden mag, so sehr wird deutlich, dass er nur schwer verfassungsrechtlich beanstandet werden konnte. ${ }^{73}$

Fraglich ist natürlich, warum das BVerfG sich überhaupt dazu entschied und beispielsweise nicht „den leichten Ausweg eines Nachwahlverbots"74 gegangen ist. Schließlich handelt es sich bei dem Effekt des NSG letztlich um eine hypothetische Wirkung mit begrenzter Reichweite, die sich nur bei Nachwahlen (mit noch weiter eingeschränkter Reichweite) instrumentalisieren lässt. ${ }^{75}$ Zudem hat jeder Eingriff in das Wahlrecht zur Behebung des Effekts des NSG Nebenwirkungen ${ }^{76}$, die zum Teil (jedenfalls verfassungspolitisch) problematischer sind als der Effekt selbst. ${ }^{77}$ Dies gilt auch für die Alternativen, die das BVerfG als

68 So hat es den Effekt des NSG mehrfach so beschrieben, „dass ein Zuwachs an Zweitstimmen [sic] zu einem Verlust an Sitzen der Landeslisten oder ein Verlust an Zweitstimmen [sic] zu einem Zuwachs an Sitzen der Landeslisten führen kann" (BVerfG, 2 BvC 1/07, 2 BvC 7/07 vom 3. Juli 2008, Ls, Tenor Nr. 1, Rn. 76, Rn. 130, Rn. 140).

69 Vgl. Christian Hesse, a.a.O. (Fn. 46), S. 188.

70 Vgl. Joachim Behnke, Überhangmandate und negatives Stimmgewicht: Zweimannwahlkreise und andere Lösungsvorschläge, in: ZParl, 41. Jg. (2010), H. 2, S. 247 - 260.

71 Dieter Nohlen, Erfolgswertgleichheit als fixe Idee oder: Zurück zu Weimar?, in: ZParl, 40. Jg. (2009), H. 1, S. $179-195$, S. 191.

72 Ebenda, S. 190.

73 Vgl. ebenda, S. 193.

74 Franz Urban Pappi / Michael Herrmann, Überhangmandate ohne negatives Stimmgewicht: Machbarkeit, Wirkungen, Beurteilung, in: ZParl, 41. Jg. (2010), H. 2, S. 260 - 278, S. 261. Es hätte auch schon gereicht, Nachwahlen auf die Direktwahl zu beschränken (vgl. Jörn Ipsen, a.a.O. (Fn. 5), S. 269).

75 Vor dem Hintergrund ist Ipsen darin zuzustimmen, die Problematik des Effekts des NSG sei überzeichnet worden (vgl. ebenda).

76 Vgl. Daniel Lübbert, Negative Stimmgewichte bei der Bundestagswahl 2009, in: ZParl, 41. Jg. (2010), H. 2, S. $278-289$, S. 289.

77 Vgl. Gerd Strohmeier, Die schlechteste Wahlsystemreform - mit Ausnahme aller anderen, in: ZfP, 58. Jg. (2011), H. 4, S. 393 - 409, S. 396. Ipsen spricht davon, dass mit der Entscheidung des Bundesverfassungsgerichts vom 3. Juli 2008 „einem bis dahin entweder nicht erkannten oder doch vernachlässigten Randphänomen des Wahlsystems eine Bedeutung zugemessen wurde, die eine Neuordnung des Wahlrechts schlechthin zur Folge haben musste" (Jörn Ipsen, a.a.O. (Fn. 5), S. 273). 
Möglichkeiten für eine Wahlrechtsreform ins Spiel gebracht hat, zum Beispiel den „Verzicht auf Listenverbindungen "78, der allerdings - wie das Gericht offensichtlich nicht bemerkt hat - nur dann den verfassungswidrigen Zustand beseitigt, wenn die Länder in abgeschlossene Wahlgebiete eingeteilt werden. ${ }^{79}$ Dabei wird deutlich, dass es - wie die Richter auch erkannt haben - „im Hinblick auf die Komplexität der zu regelnden Materie [...] nicht in Betracht“ kam, „dass das Bundesverfassungsgericht [selbst] die rechtliche Grundlage für die Durchführung der Wahlen bereit stellt" ${ }^{\text {" }}$. Davon abgesehen hat das BVerfG den Handlungsspielraum des Gesetzgebers ohnehin schon stark eingeschränkt - dem, solange er an der Grundstruktur der personalisierten Verhältniswahl festhalten wollte, nur ein äußerst geringer Spielraum zu deren Ausgestaltung blieb. Die nachfolgenden Wahlrechtsdebatten und -reformen haben auch gezeigt, dass es nur wenige Alternativen für eine verfassungskonforme Regelung der bestehenden personalisierten Verhältniswahl gibt und letztlich jede davon ungewünschte Nebenwirkungen hat. ${ }^{81}$

Etwas widersprüchlich mutet an, dass das BVerfG in seinem Urteil vom 3. Juli 2008 einerseits zu dem Ergebnis kam, dass der Effekt des NSG ,in eklatanter Weise“ ${ }^{82}$ gegen den Grundsatz der Gleichheit der Wahl verstoße, andererseits aber feststellte, dass „der Wahlfehler nicht so gewichtig [sei], dass er die Auflösung des 16. Deutschen Bundestages rechtfertigen würde" 83 , und dem Gesetzgeber bis zum 30. Juni 2011, also bis nach der Bundestagswahl 2009, Zeit gab, um den verfassungswidrigen Zustand zu beheben. ${ }^{84}$ Dennoch hat dieser erst am 29. September 2011 über ein neues Bundeswahlgesetz entschieden, das am 3. Dezember 2011 in Kraft getreten ist und gegen das erneut geklagt wurde.

Am 25. Juli 2012 hat das BVerfG über diese Klage entschieden und dabei unter anderem festgestellt, dass die mit der Wahlrechtsreform 2011 eingeführte Bestimmung der Sitzkontingente der Länder nach der Wählerzahl „die Grundsätze der Gleichheit und Unmittelbarkeit der Wahl sowie der Chancengleichheit der Parteien "85 verletze, da sie den Effekt des NSG „nicht nur in seltenen, vernachlässigbaren Ausnahmefällen “86, sondern „mit beachtlicher Wahrscheinlichkeit“ - „mindestens in etwa der gleichen Größenordnung [...] wie [...] im bisherigen Wahlrecht" 87 - ermögliche. Sowohl die festgestellte Generierung des Effekts

78 BVerfG, 2 BvC 1/07, 2 BvC 7/07 vom 3. Juli 2008, Rn. 124.

79 Vgl. Hans Meyer, in: A-Drs. 16(4)592 B, S. 4.

80 BVerfG, 2 BvC 1/07, 2 BvC 7/07 vom 3. Juli 2008, Rn. 138.

81 Vgl. dazu exemplarisch die Debatte in der ZParl: Joachim Behnke, a.a.O. (Fn. 70); Franz Urban Pappi / Michael Herrmann, a.a.O. (Fn. 74); Daniel Lübbert, a.a.O. (Fn. 76); Gerd Strohmeier, Die Geister, die Karlsruhe rief - Eine Replik auf die Beiträge zur Wahlsystemreform in Heft 2/2010 der ZParl, in: ZParl, 42. Jg. (2011), H. 1, S. 186 - 193; Joachim Behnke / Florian Grotz, Das Wahlsystem zwischen normativer Begründung, empirischer Evidenz und politischen Interessen. Ein Kommentar zu Gerd Strohmeier sowie Franz Urban Pappi und Michael Herrmann, in: ZParl, 42. Jg. (2011), H. 2, S. 419 - 425; Joachim Behnke, Ein sparsames länderproporzoptimierendes parteiproporzgewährendes automatisches Mandatszuteilungsverfahren mit Ausgleich ohne negatives Stimmgewicht, in: ZParl, 43. Jg. (2012), H. 3, S. 675 - 693; Christian Hesse, a.a.O. (Fn. 46); Niels Dehmel/ Eckhard Jesse, a.a.O. (Fn. 48).

82 BVerfG, 2 BvC 1/07, 2 BvC 7/07 vom 3. Juli 2008, Rn. 103.

83 Ebenda, Rn. 138.

84 Vgl. ebenda, Rn. 144.

85 BVerfG, 2 BvC 1/11, 2 BvC 2/11 vom 4. Juli 2012, Rn. 66.

86 Ebenda, Rn. 94.

87 Ebenda, Rn. 95. 
an sich als auch das skizzierte Ausmaß können (zumindest auf der Grundlage des Gerichtsurteils vom 3. Juli 2008) mit gewichtigen Argumenten, die an anderer Stelle entfaltet wur$\operatorname{den}^{88}$, in Zweifel gezogen werden. Jedenfalls wird dabei deutlich, dass die Karlsruher Richter den NSG-Tatbestand „ausgedehnt "89 und dabei den Gestaltungsspielraum des Gesetzgebers weiter eingeschränkt haben. ${ }^{90}$ Darüber hinaus führten sie erneut nicht nur den klassischen NSG-Tatbestand auf („dass ein Zuwachs an Stimmen zu Mandatsverlusten führt“ beziehungsweise „dass für den Wahlvorschlag einer Partei insgesamt mehr Mandate erzielt werden, wenn auf ihn selbst weniger [...] Stimmen entfallen“), sondern auch den erweiterten NSG-Tatbestand („dass für den Wahlvorschlag einer Partei insgesamt mehr Mandate erzielt werden, wenn [...] auf einen konkurrierenden Vorschlag mehr Stimmen entfallen"91). Zudem wird im Gegensatz zum Urteil vom 3. Juli 2008, in dem bei der Definition des Effekts des NSG - wie oben ausgeführt - relativ klar auf die Zweitstimmen abgestellt wurde, an zentraler Stelle nur noch von Stimmen gesprochen ${ }^{92}-$ mit der Folge neuerlicher Interpretationsbedürftigkeit. Hinzu kommt, dass die Aussage, der durch Überhangmandate „gestörte Proporz [könnte] durch Zuteilung von Ausgleichsmandaten wiederhergestellt werden "93, widersprüchlich oder zumindest unpräzise ist, da ein Ausgleich von Überhangmandaten offenkundig zwangsläufig zu NSG, zumindest zu dem erweiterten NSG-Tatbestand, führt. So erhalten andere Parteien mehr Ausgleichsmandate, wenn eine Partei mehr Erststimmen und in der Folge mehr Überhangmandate bekommt. Aufgrund des widersprüchlichen beziehungsweise unpräzisen Urteils war der Gesetzgeber gezwungen, „[e]ine Interpretation der Definition des negativen Stimmgewichts" ${ }^{44}$ durch das Gericht vorzunehmen, nach der mit der Vergabe von Ausgleichsmandaten zwangsläufig verbundene NSG-Effekte nicht verfassungswidrig sind beziehungsweise diese Effekte nicht als (verfassungsgerichtlich beanstandete) NSG-Effekte betrachtet werden. Erstaunlich ist im Übrigen auch, dass das BVerfG bei der Bestimmung der Sitzkontingente der Länder nach der Wählerzahl den Effekt des NSG feststellte, obwohl dies - wie oben angemerkt - durchaus strittig ist, und bei der Reststimmenverwertung beziehungsweise der Vergabe der Zusatzmandate

88 Vgl. hierzu ausführlich Gerd Strohmeier, a.a.O. (Fn. 77), S. 397 ff.; Volker M. Haug, a.a.O. (Fn. 21), S. 662 ff.; Christian Hesse, a.a.O. (Fn. 46), S.180 ff., S. 187 ff.

89 Schließlich könne das Sitzzuteilungsverfahren „infolge der Bildung der Ländersitzkontingente nach der Wählerzahl [sic] dazu führen, dass in bestimmten Konstellationen [sic] abgegebene Zweitstimmen für Landeslisten einer Partei insofern negativ wirken, als diese Partei in einem anderen Land Mandate verliert oder eine andere Partei Mandate gewinnt. Umgekehrt ist es auch möglich, dass die Nichtabgabe [sic] einer Wählerstimme der zu unterstützenden Partei dienlich ist. Dieser [sic] Effekt des negativen Stimmgewichts ist verfassungsrechtlich nicht gerechtfertigt" (BVerfG, 2 BvF 3/11, 2 BvR 2670/11, 2 BvE 9/11 vom 25. Juli 2012, Rn. 86). So hätte Die Linke bei der Bundestagswahl 2009 ,insgesamt ein Mandat mehr erzielt, wenn auf ihre Landesliste in Bayern eine bestimmte Zahl von Zweitstimmen weniger entfallen wäre, weil [sic] diese Wähler keinen Stimmzettel abgegeben hätten“ (ebenda, Rn. 88). Schließlich hänge „der Effekt des negativen Stimmgewichts davon ab, dass mit der Veränderung der Zweitstimmenzahl in einem Land eine Veränderung der Wählerzahl [sic] einhergeht und dadurch eine Sitzverschiebung zwischen den Ländern bewirkt wird“ (ebenda, Rn. 96).

90 Vgl. Volker M. Haug, a.a.O. (Fn. 21), S. 665.

91 BVerfG, 2 BvF 3/11, 2 BvR 2670/11, 2 BvE 9/11 vom 25. Juli 2012, Rn. 85.

92 Vgl. ebenda.

93 Ebenda, Rn. 135.

94 BT-Drs. 17/11819, S. 6. 
den Effekt nicht feststellte, obwohl dessen Eintreten in diesem Bereich - wenn auch nur in seltenen, vernachlässigbaren Ausnahmefällen - völlig unstrittig ist. ${ }^{95}$

\section{Wablrecht von Auslandsdeutschen}

Nach dem vom Bundesverfassungsgericht in seinem Urteil vom 4. Juli 2012 beanstandeten $\$ 12$ Absatz 2 Satz 1 BWG waren diejenigen Deutschen (sofern sie die sonstigen Voraussetzungen erfüllten) wahlberechtigt, „die am Wahltag außerhalb der Bundesrepublik Deutschland leben, sofern sie nach dem 23. Mai 1949 und vor ihrem Fortzug mindestens drei Monate ununterbrochen in der Bundesrepublik Deutschland eine Wohnung innegehabt oder sich sonst gewöhnlich aufgehalten haben“. Dabei ist darauf hinzuweisen, dass der Gesetzgeber in der Vergangenheit „die weiteren Voraussetzungen für das aktive Wahlrecht (neben einem früheren dreimonatigen Inlandsaufenthalt) schrittweise aufgehoben [hat], um nicht gerechtfertigte Benachteiligungen einzelner Gruppen von Auslandsdeutschen zu beseitigen“, und das BVerfG die vom Gesetzgeber eingeschlagene Zielrichtung „mehrfach für mit der Verfassung vereinbar erklärt" ${ }^{" 96}$ hat. So hat es in seinem Beschluss vom 2. November 1990 die damalige Regelung, die sich nicht fundamental beziehungsweise nur partiell von der 2012 verworfenen unterschied, nicht beanstandet. ${ }^{97}$ Die betreffende Regelung „kombinierte das Erfordernis dreimonatiger Sesshaftigkeit mit einer Fortzugsfrist von maximal zehn Jahren, wobei zugunsten von in Europaratsstaaten Ansässigen auf die Fortzugsfrist verzichtet wurde“98. Folglich galt für die Auslandsdeutschen - mit Ausnahme der nicht in Mitgliedstaaten des Europarats Ansässigen - die 2012 für verfassungswidrig erklärte Regelung. Zudem ist die später - durch Streichung der Fortzugsfrist bei außerhalb von Europarats-Staaten Ansässigen - erfolgte Vereinheitlichung der Regelung im Urteil vom 4. Juli 2012 nicht beanstandet worden.

Allerdings entschied das Bundesverfassungsgericht am 4. Juli 2012, dass „\$1 12 Absatz 2 Satz 1 des Bundeswahlgesetzes in der Fassung des Gesetzes zur Änderung des Wahl- und Abgeordnetenrechts vom 17. März 2008 [...] mit Artikel 38 Absatz 1 Satz 1 des Grundgesetzes unvereinbar und nichtig“99 ist. Dabei führte es aus, dass zu den Gründen, „die Differenzierungen im Anwendungsbereich des Grundsatzes der Allgemeinheit der Wahl rechtfertigen können“, „insbesondere die mit demokratischen Wahlen verfolgten Ziele der Sicherung des Charakters der Wahl als eines Integrationsvorgangs bei der politischen Willensbildung des Volkes“ zähle, wozu „die Sicherung der so genannten Kommunikationsfunktion der Wahl“ ${ }^{100}$ gehöre. Infolgedessen stehe „es dem Gesetzgeber frei, zur Sicherung der Kommunikationsfunktion der Wahl zu verlangen, dass im Ausland lebende Deutsche imstande sind, am Kommunikationsprozess zwischen Volk und Staatsorganen teilzunehmen“, und sei es „verfassungsrechtlich zulässig, für das aktive Wahlrecht ein Mindestmaß an persönlich und

95 Vgl. Gerd Strohmeier, a.a.O. (Fn. 77), S. 400 f.; Christian Hesse, a.a.O. (Fn. 46), S. 188 ff.

96 BT-Drs. 17/11820, S. 3.

97 Vgl. BVerfG, 2 BvR 1266/90 vom 2. November 1990.

98 BVerfG, 2 BvC 1/11, 2 BvC 2/11 vom 4. Juli 2012, Abweichende Meinung der Richterin Gertrude Lübbe-Wolff, Rn. 67.

99 BVerfG, 2 BvC 1/11, 2 BvC 2/11 vom 4. Juli 2012, Tenor Nr. 2.

100 Ebenda, Rn. 39. 
unmittelbar erworbener Vertrautheit mit dem politischen System der Bundesrepublik Deutschland vorauszusetzen“"101. Allerdings verstoße „[d]ie Anknüpfung der Wahlberechtigung der Auslandsdeutschen allein an einen früheren dreimonatigen Daueraufenthalt im Bundesgebiet " 102 gegen den Grundsatz der Allgemeinheit der Wahl. Angesichts der oben aufgeführten früheren Verfassungsrechtsprechung zum Wahlrecht von Auslandsdeutschen kann das Urteil vom 4. Juli 2012 - wie die Urteile vom 3. Juli 2008 und 25. Juli 2012 - nur überraschen und als Kehrtwende bezeichnet werden. Niemand brachte dies so gut zum Ausdruck wie die Richterin Gertrude Lübbe-Wolff in ihrer abweichenden Meinung zum Beschluss des Zweiten Senats vom 4. Juli 2012: „Sollten die am Gesetzgebungsverfahren Beteiligten gemeint haben, dass man sich an ständiger Rechtsprechung des Bundesverfassungsgerichts zumindest dann gefahrlos orientieren kann, wenn nichts dafür ersichtlich ist, dass sie innerhalb des Gerichts jemals umstritten gewesen wäre, muss der vorliegende Beschluss sie überraschen." 103

Im Kern hat das BVerfG entschieden, dass $\$ 12$ Absatz 2 Satz 1 BWG weder geeignet ist, einen Teil der Auslandsdeutschen, die nach dem Normzweck von der Wahlteilnahme ausgeschlossen werden sollten, von der Wahlteilnahme auszuschließen, noch jenem Teil der Auslandsdeutschen, denen nach dem Normzweck die Wahlteilnahme ermöglicht werden sollte, die Wahlteilnahme zu ermöglichen.

Zum einen könne „[d]as erklärte Ziel des Gesetzgebers, die für die Wahlteilnahme vorauszusetzende Vertrautheit mit den politischen Verhältnissen in der Bundesrepublik Deutschland zu sichern, [...] allein mit dem Erfordernis eines früheren dreimonatigen Aufenthalts in Deutschland nicht erreicht werden" ${ }^{104}$. So schließe die Regelung eine nicht vernachlässigbare Zahl Auslandsdeutscher nicht von der Wahlteilnahme aus, obwohl diese mit Blick auf den Normzweck ausgeschlossen werden müssten. Dies betreffe zum einen diejenigen, „die sich zu einem Zeitraum in der Bundesrepublik Deutschland aufgehalten haben, zu dem sie die notwendige Vertrautheit mit den hiesigen politischen Verhältnissen mangels hinreichender Reife und Einsichtsfähigkeit nicht erwerben konnten“, und zum anderen diejenigen, „die die Bundesrepublik Deutschland schon vor so langer Zeit verlassen haben, dass die von ihnen erworbenen eigenen Erfahrungen in den aktuellen politischen Verhältnissen keine Entsprechung mehr finden "105. Den Ausschluss der ersten Teilgruppe von der Wahlteilnahme könne der Gesetzgeber „durch die Aufnahme einer zusätzlichen Altersgrenze" erreichen; den Ausschluss der zweiten Teilgruppe durch die Festlegung einer „angemessene[n] Fortzugsfrist“, wobei das Gericht auf frühere Fassungen des $\$ 12$ Abs. 2 Satz 1 BWG verwies. ${ }^{106}$

Zum anderen bewirke das Erfordernis eines früheren dreimonatigen Aufenthalts in Deutschland „entgegen der Gesetzesintention, dass Deutsche, die typischerweise mit den politischen Verhältnissen vertraut und von ihnen betroffen sind, an den Wahlen zum Deutschen Bundestag nicht teilnehmen können" ${ }^{107}$. Folglich schließe die Regelung Auslands-

101 BT-Drs. 17/11820, S. 3; vgl. BVerfG, 2 BvC 1/11, 2 BvC 2/11 vom 4. Juli 2012, Rn. 47 ff.

102 Ebenda, Ls 2.

103 BVerfG, 2 BvC 1/11, 2 BvC 2/11 vom 4. Juli 2012, Abweichende Meinung der Richterin Gertrude Lübbe-Wolff, Rn. 66.

104 BVerfG, 2 BvC 1/11, 2 BvC 2/11 vom 4. Juli 2012, Rn. 50.

105 Ebenda, Rn. 52.

106 Ebenda, Rn. 54.

107 Ebenda, Rn. 50. 
deutsche von der Wahlteilnahme aus, obwohl diese mit Blick auf den Normzweck nicht ausgeschlossen werden dürften. Dies betreffe diejenigen, „die zwar zu keinem Zeitpunkt für mindestens drei Monate in der Bundesrepublik Deutschland ansässig gewesen sind, jedoch typischerweise mit den politischen Verhältnissen vertraut und von ihnen betroffen sind, etwa weil sie als ,Grenzgänger' ihre Berufstätigkeit in der Bundesrepublik Deutschland ausüben [...] oder weil sie durch ihr Engagement in Verbänden, Parteien und sonstigen Organisationen in erheblichem Umfang am politischen und gesellschaftlichen Leben der Bundesrepublik Deutschland teilnehmen“108. Im Übrigen sei „[d] ie Einbeziehung dieser Teilgruppe der Auslandsdeutschen in den Kreis der Wahlberechtigten [...] selbst dann, wenn der Gesetzgeber an seiner Grundentscheidung festhält, möglich"109.

Dabei wird deutlich, dass der Gesetzgeber, solange er an seiner Grundentscheidung festhält, nur einen äußerst geringen Spielraum zur Ausgestaltung dieser Grundentscheidung hat beziehungsweise für diesen (äußerst wahrscheinlichen) Fall das BVerfG eine mustergültige Lösung entworfen hat, von der er kaum abweichen kann.

Die Karlsruher Richter begründeten ihre Kehrtwende im Urteil vom 4. Juli 2012 damit, dass „das - mehrfach geänderte - Wahlrecht der Auslandsdeutschen nicht ohne Blick auf die jeweiligen rechtlichen und tatsächlichen Rahmenbedingungen verfassungsrechtlich gewürdigt werden“ könne und „Aussagen des Bundesverfassungsgerichts zu früheren Ausgestaltungen der Wahlberechtigung der Auslandsdeutschen [...] daher nicht ohne Weiteres zur Beurteilung der aktuellen Rechtslage herangezogen werden " ${ }^{110}$ könnten. Fraglich ist allerdings, inwiefern sich die „rechtlichen und tatsächlichen Rahmenbedingungen“ seither geändert haben und Diskrepanzen erkennen lassen. Zweifelsohne „hat die Anknüpfung des Wahlrechts an einen aktuell bestehenden oder nur wenige Jahre zurückliegenden mindestens dreimonatigen Wohnsitz oder gewöhnlichen Aufenthalt im Wahlgebiet in den zurückliegenden Jahrzehnten durch die Entwicklung von Mobilität und Kommunikationstechnik an Plausibilität eingebüßt"111. Allerdings hat der Gesetzgeber auf diese Entwicklung reagiert und die bestehenden Wahlrechtsbeschränkungen für Auslandsdeutsche sukzessive abgebaut: „Er hat zunächst einen Zeitraum, um den die geforderte dreimonatige Präsenz zurückliegen darf, eingeführt, diese sogenannte Fortzugsfrist später von zehn auf fünfundzwanzig Jahre verlängert und sie schließlich ganz abgeschafft. Zwischenzeitliche gruppenbezogene Differenzierungen wie die Privilegierung der in Europaratsstaaten ansässigen Auslandsdeutschen [...] sind im Zuge dieser Rechtsentwicklung entfallen. "112 Abgesehen davon kann die Berücksichtigung der tatsächlichen Rahmenbedingungen, namentlich die Verbesserung der Kommunikationsmöglichkeiten (insbesondere über das Internet), und die Tatsache, dass das Bundesverfassungsgericht die „Kommunikationsfunktion der Wahl [...] als möglichen Rechtfertigungsgrund für Beschränkungen des Wahlrechts Auslandsdeutscher in den Vordergrund" 113 gestellt hat, eigentlich nur zu der Einsicht führen, dass die bestehende Regelung entschärft, aber nicht verschärft werden müsse.

108 Ebenda, Rn. 56.

109 Ebenda.

110 Ebenda, Rn. 38.

111 BVerfG, 2 BvC 1/11, 2 BvC 2/11 vom 4. Juli 2012, Abweichende Meinung der Richterin Gertrude Lübbe-Wolff, Rn. 70.

112 Ebenda.

113 Ebenda, Rn. 68. 
Vor diesem Hintergrund kann es nur verwundern, dass das BVerfG „den sehr viel weiterreichenden Ausschluss vom Wahlrecht durch eine Kombination dieser Lösung [der bestehenden Regelung] mit einer Fortzugsfrist billigt"114. Die Tatsache, dass das Gericht einerseits eine Verschärfung der bestehenden Rechtslage, andererseits aber Ausnahmen davon in Form einer Sonderregelung fordert, verstärkt diese Verwunderung noch. Schließlich ignoriert das Gericht dabei, was die dissentierende Richterin Lübbe-Wolffins Urteil schrieb, dass „[j]ede praktikable Lösung [...] unvermeidbar mit der Misslichkeit verbunden [ist], dass sie entweder fragwürdige Einschlüsse oder fragwürdige Ausschlüsse oder - in jeweils weniger fragwürdigem Ausmaß - beides produziert"115. Zudem wurde vom BVerfG nicht berücksichtigt, dass ein Teil derer, die durch die Sonderregelung erfasst werden sollen, bereits unter die „Dreimonatsregel“ fallen. So trägt die bestehende Rechtslage „[d]en Inlandsbindungen des typischen Grenzpendlers, der sich irgendwann aus finanziellen oder sonstigen Gründen dafür entschieden hat, jenseits der Grenze zu wohnen, [...] bereits Rechnung “116. Ob der andere Teil, der nicht unter die „Dreimonatsregel“ fällt, „in irgendeinem möglichen Wortsinn ,typischerweise“ auftritt“", ist fragwürdig; ob er, selbst wenn dem so wäre, zwingend zu berücksichtigen ist, ebenfalls, „weil dieser Typus sich nicht [oder kaum] mit praktikablen Abgrenzungskriterien erfassen lässt" "117.

Fraglich ist auch, inwiefern die „Dreimonatsregel“ überhaupt ein Kriterium für die „Kommunikationsfunktion der Wahl“118 sein kann. In der Tat „mag das wenig einleuchten“, zumal auch der Gesetzgeber die Dreimonatsregel nicht „[a]ls Kriterium für wahlrechtsrelevantes Kommunikationspotential“, sondern als Kriterium für „ein Mindestmaß an realer Verbindung zur Bundesrepublik Deutschland“ gesehen hat, wodurch ein Ausgleich zwischen entgegengesetzten verfassungsrechtlichen Belangen geschaffen wird, der „bislang noch nirgends als verfassungswidrig beurteilt worden ist" ${ }^{119}$. Daran schließt die Frage an, warum das BVerfG den Kommunikationszusammenhang in den Mittelpunkt rückt, da „nicht der Kommunikationszusammenhang, sondern der Verantwortungszusammenhang der grundlegendere" 120 ist. Dies wurde in der abweichenden Meinung der Richterin Gertrude Lübbe-Wolff zu Recht herausgestellt: „Je öfter und weiter formelle Zugehörigkeit [...] und materielle Betroffenheit von der Staatsgewalt, auf die mit der Wahlentscheidung Einfluss genommen wird, auseinanderfallen, desto mehr entspricht es daher dem Sinn demokratischer Wahlen, die Wahlberechtigung nicht allein an die formelle Zugehörigkeit, sondern darüber hinaus daran zu knüpfen, dass die Wählenden mit ihrer Wahlentscheidung auf die politische Gestaltung eigener, nicht fremder, Lebensverhältnisse Einfluss nehmen. "121 Vor diesem Hintergrund hat der Gesetzgeber in der Vergangenheit auf das Erfordernis des mindestens dreimonatigen Aufenthalts zurückgegriffen - und das Bundesverfassungsgericht dies bis zu seinem Urteil vom 4. Juli 2012 gebilligt. ${ }^{122}$

114 Ebenda, Rn. 75.

115 Ebenda, Rn. 74.

116 Ebenda, Rn. 76.

117 Ebenda (mit weiteren Argumenten).

118 Ebenda, Rn. 68.

119 Ebenda, Rn. 70 (mit weiteren Nachweisen).

120 Ebenda, Rn. 73.

121 Ebenda, Rn. 73 (mit weiteren Nachweisen).

122 Vgl. ebenda (mit weiteren Nachweisen). 


\section{Von Richtern zu Politikern}

Für den, der die Rechtsprechung des Bundesverfassungsgerichts im Bereich des Wahlrechts - konkret: zu Überhangmandaten, zum Effekt des negativen Stimmgewichts und zum Wahlrecht von Auslandsdeutschen - kennt, ist die Frage, ob sich der Gesetzgeber auf das Gericht in Karlsruhe verlassen könne, rhetorisch - und aus guten Gründen mit Nein zu beantworten. In allen drei Bereichen hat es in den letzten Jahren beinahe artistische Kehrtwenden vollzogen: Während das BVerfG in seinem Urteil vom 10. April 1997 feststellte, dass primär der Gesetzgeber zu entscheiden habe, in welchem Umfang Überhangmandate hinzunehmen seien, und für diese Entscheidung das Fünf-Prozent-Quorum einen „Orientierungspunkt“ biete, hat es in seinem Urteil vom 25. Juli 2012 dieses Quorum halbiert und als verbindliche „Obergrenze“ definiert; während es in seinem Urteil vom 3. Juli 2008 feststellte, dass der Effekt des NSG „in eklatanter Weise“123 gegen den Grundsatz der Gleichheit der Wahl verstoße, hat es „zuvor - [...] in voller Kenntnis des bereits im Jahre 1997 in dem von der Landesregierung Niedersachsen geführten Normenkontrollverfahren gerügten und thematisierten NSG - entschieden beziehungsweise dem Bundestag in Berichterstatterschreiben signalisiert [...], dass die NSG verfassungskonform seien“124; während es in seinem Urteil vom 4. Juli 2012 feststellte, dass „[d]ie Anknüpfung der Wahlberechtigung der Auslandsdeutschen allein an einen früheren dreimonatigen Daueraufenthalt im Bundesgebiet" gegen den Grundsatz der Allgemeinheit der Wahl verstoße, hatte es in seinem Beschluss vom 2. November 1990 die damalige, beinahe identische Regelung nicht beanstandet. Insgesamt ist der Einschätzung Ipsens zuzustimmen, „dass die Rechtsprechung des BVerfG keineswegs geradlinig verlaufen ist und für den Gesetzgeber deshalb nicht immer hilfreich war"125. Natürlich gibt es im Zeitverlauf nicht das Bundesverfassungsgericht: Die Zusammensetzung des Gerichts war am 25. Juli 2012 eine andere als am 10. April 1997. Und natürlich kann eine Veränderung der Rahmenbedingungen zu einer veränderten verfassungsrechtlichen Bewertung führen, die, wenn sie entsprechend begründet ist, auch inhaltlich akzeptiert werden kann.

Die Kehrtwenden, die das Bundesverfassungsgericht in den oben aufgeführten Bereichen vollzogen hat, wurden allerdings keineswegs überzeugend oder nur durch eine gewisse „Argumentationsakrobatik“126 beziehungsweise spezielle „Norminterpretationskunst“127 begründet. Dabei ist die Begründung einer Verfassungsgerichtsentscheidung, gerade bei einem offenen Bruch „mit der bisherigen Rechtsprechungslinie in einer politisch bedeutsamen und verfassungsrechtlich sensiblen Frage" 128 , von fundamentaler Bedeutung für die Akzeptanz dieser Entscheidung - und in der Folge für die Akzeptanz des Gerichts. Grundsätzlich gilt: „Je überzeugender eine Entscheidung begründet ist, umso mehr wird sie in Wissenschaft und politischer Öffentlichkeit Anerkennung finden und als das durch die Verfassung ,Gewollte' erscheinen. " ${ }^{129}$ Doch weder beim Wahlrecht von Auslandsdeutschen

123 BVerfG, 2 BvC 1/07, 2 BvC 7/07 vom 3. Juli 2008, Rn. 103.

124 Heinrich Lang, in: A-Drs. 16(4)592 F, S. 3.

125 Jörn Ipsen, a.a.O. (Fn. 5), S. 265.

126 Dieter Nohlen, a.a.O. (Fn. 71), S. 193.

127 Volker M. Haug, a.a.O. (Fn. 21), S. 671.

128 Ebenda, S. 670.

129 Jörn Ipsen, a.a.O. (Fn. 5), S. 273. 
noch in der Frage der Überhangmandate oder beim Effekt des NSG wurde die vollzogene Kehrtwende überzeugend begründet. In keinem dieser Fälle lässt sich eine Neubewertung des jeweiligen Sachverhalts aufgrund spezifischer Veränderungen der „jeweiligen rechtlichen und tatsächlichen Rahmenbedingungen "130 rechtfertigen, da sich weder das eine noch das andere - in für die jeweilige Entscheidung relevanter Weise - verändert hat. Infolgedessen fehlte es den Urteilsbegründungen am 3. Juli 2008, 4. Juli 2012 und 25. Juli 2012 an Überzeugungskraft. Sie waren in Teilen widersprüchlich, unpräzise oder unzutreffend. Dies gilt für die generelle Kehrtwende ebenso wie für die konkrete Festlegung der (unpräzise angegebenen) neuen „Obergrenze“ bei Überhangmandaten im Urteil vom 25. Juli 2012, für die festgestellte Verletzung der Gleichheit und der Freiheit der Wahl durch den Effekt des NSG im Urteil vom 3. Juli 2008 sowie für die konstatierte Notwendigkeit, beim Wahlrecht von Auslandsdeutschen die „Dreimonatsregel“ einzuschränken und eine Sonderregelung zu schaffen, im Urteil vom 4. Juli 2012. Besonders problematisch ist natürlich, wenn das Bundesverfassungsgericht selbst einräumt, dass ein „Akt richterlicher Normkonkretisierung nicht vollständig begründet werden kann“131. Schließlich hat Haug zu Recht festgestellt: „Was juristisch nicht begründet werden kann, ist letztlich politisch motiviert." ${ }^{132}$

Offenbar hat sich das Gericht zunehmend von seinem sich selbst auferlegten Grundsatz des judicial restraint entfernt - also von dem „Verzicht, Politik zu treiben', das heißt in den von der Verfassung geschaffenen und begrenzten Raum freier politischer Gestaltung einzugreifen“ beziehungsweise „den von der Verfassung für die anderen Verfassungsorgane garantierten Raum freier politischer Gestaltung offenzuhalten“133. Spätestens dann, wenn renommierte Medien wie Die Zeit die Frage stellen „Richter oder Politiker?"134, scheint der Grat zwischen verfassungsrechtlicher Kontrolle und politischer Dezision allmählich überschritten zu sein.

130 BVerfG, 2 BvC 1/11, 2 BvC 2/11 vom 4. Juli 2012, Rn. 38.

131 BVerfG, 2 BvF 3/11, 2 BvR 2670/11, 2 BvE 9/11 vom 25. Juli 2012, Rn. 144.

132 Volker M. Haug, a.a.O. (Fn. 21), S. 671.

133 BVerfG, 2 BvF 1/73 vom 31. Juli 1973, Rn. 75.

134 Heinrich Wefing, Richter oder Politiker?, in: Die Zeit vom 2. Mai 2013, S. 2. 